\title{
Common Knowledge and Consensus with Noisy Communication ${ }^{1}$
}

\author{
Frédéric KoessLeR \\ BETA - Theme \\ Université Louis Pasteur, 61 Avenue de la Forêt-Noire \\ F-67085 Strasbourg Cedex (France) \\ E-mail: koessler@cournot.u-strasbg.fr
}

March 23, 2000

\footnotetext{
${ }^{1}$ I am deeply indebted to Gaël Giraud for helpful comments and suggestions. I am grateful to Hubert Stahn, Gisèle Umbhauer, and particularly Guillaume Haeringer for help in improving the exposition of the paper. I also thank Patrick Roger and Anthony Ziegelmeyer for helpful conversations during a seminar presentation. Of course, any remaining errors are my own responsibility.
} 


\begin{abstract}
Parikh and Krasucki (1990) suggested in an informal manner that a consensus does not require common knowledge. Weyers (1992) proved that their model does not permit such a conclusion and that a more general one has to be constructed. Heifetz (1996) gave an example with three agents inspired by computer science which illustrates the intuition of Parikh and Krasucki (1990), i.e., where a consensus is obtained without common knowledge of it. We propose a general setting of noisy communication to confirm this result. We show that common knowledge cannot emerge with any non-public and noisy communication protocol. But, with "fair" protocols and a sufficiently rich language, a consensus and arbitrary high levels of interactive knowledge are achievable. A minimal example with two agents and two states is given. Nevertheless, for public and noisy communication, some results on common knowledge and consensus are obtained. We apply our results to describe some conditions that ensure or prevent epistemic conditions for Nash equilibrium. In general, non-public and noisy communication is not sufficient for the conjectures to form, during time, a Nash equilibrium, even if the game and mutual rationality are mutually known. However, with only two agents or with a noisy and public communication protocol, sufficient conditions are given for the conjectures to form a Nash equilibrium in a finite number of communication periods.
\end{abstract}

KEYwords: Noisy communication protocols, common knowledge, consensus, Nash equilibrium, conjectures.

JEL Classification: C72, D82. 


\section{Résumé}

Parikh et Krasucki (1990) ont suggéré de manière informelle qu'un consensus ne nécessite pas la connaissance commune. Weyers (1992) a prouvé que leur modélisation ne permet pas d'obtenir une telle conclusion, et qu'un modèle plus général doit donc être construit. Heifetz (1996) a donné un exemple à trois agents, inspiré par les travaux effectués en informatique théorique, qui illustre l'intuition de Parikh et Krasucki (1990), i.e., où un consensus est atteint sans qu'il ne devienne connaissance commune. Nous proposons un cadre général qui confirme ce résultat. Nous montrons que pour tout protocole de communication faillible et non public, aucun événement ne peut devenir connaissance commune s'il ne l'était pas au départ. Cependant, si le langage disponible est suffisamment riche, un consensus et un niveau arbitraire de connaissances interactives peuvent être obtenus avec des protocoles "fair". Un exemple minimal à deux agents et deux états de la nature est donné. Pour des protocoles de communication faillibles mais publics, nous proposons des résultats à la fois sur l'évolution de la connaissance commune et l'obtention de consensus. Nous appliquons nos résultats afin de décrire des conditions qui assurent ou empêchent d'obtenir les conditions épistémiques de l'équilibre de Nash. En général, la communication faillible et non publique n'est pas suffisante à l'obtention de conjectures formant un équilibre de Nash, même si la rationalité et le jeu sont une connaissance mutuelle. Cependant, avec uniquement deux agents, ou si la communication est publique, des conditions suffisantes assurant un équilibre de Nash en un nombre fini de périodes sont proposées.

Mots Clés : Communication faillible, protocoles, connaissance commune, consensus, équilibre de Nash, conjectures.

Classification JEL : C72, D82. 


\section{Introduction}

When individuals with different knowledge communicate some messages to each other, each one integrates in his own knowledge the information which is contained in the messages he received. But each individual also takes into account others' knowledge about this information. In the same way, each individual also considers his own knowledge about others' knowledge about this information, and so on. When communication takes place, all these hierarchies of knowledge are incorporated into individuals' knowledge. Thus, the learning process that occurs during communication involves not only first-order knowledge but also higher-order knowledge. In other words, when some information is transferred from a set of senders to a set of receivers, every agent learns something because interactive knowledge (i.e., knowledge about knowledge) is also modified. When iterative reasoning about interactive knowledge is applied infinitely many times and leads to the same knowledge for every individual, this knowledge is called common knowledge. More precisely, an event is said to be common knowledge if everyone knows it, everyone knows that everyone knows it, everyone knows that everyone knows that everyone knows it, and so on ad infinitum.

A fundamental phenomenon related to common knowledge is consensus (or agreement). When agents can communicate their beliefs, or more generally when they can communicate messages determined by the value of a function that depends on their private information, we say that a consensus about this function is achieved when agents agree about its value. In this case, all agents send the same message, i.e., communicated values are equal. Common knowledge of these values was shown to be a sufficient condition for consensus. ${ }^{1}$ Furthermore, when a consensus emerges, common knowledge of this consensus also naturally seems to emerge. However, in some practical settings, this last conclusion remains problematic. Indeed, common knowledge is particularly difficult to achieve through communication if there are intertemporal uncertainties, i.e., if agents are uncertain about the period at which other agents' learning occurs. When communication is noisy, i.e., if some messages may fail to reach the receivers at some periods, intertemporal uncertainties appear because agents are uncertain about others' current information. Since common knowledge requires perfect transparency of interactive knowledge, communication in these conditions does not seem to create common knowledge. Nevertheless, the emergence of a consensus seems to be possible. In such a setting, the analysis of the emergence of common knowledge and consensus, and of their interdependence, is still restrained.

The aim of this paper is to formally analyze under which conditions common knowledge and consensus could emerge when there are intertemporal

\footnotetext{
${ }^{1}$ See Aumann (1976).
} 
uncertainties generated by noisy communication. We show that the type of communication protocol and the richness of the available language are crucial elements for the emergence of consensus and common knowledge. Our results can be applied in any context where the notion of common knowledge intervenes. For example, they can be used to analyze the emergence of epistemic conditions that characterize solution concepts in game theory. In the last section, such an application is done to characterize conditions ensuring or preventing epistemic conditions for Nash equilibrium ${ }^{2}$ when players' conjectures are communicated through noisy communication protocols.

The notions of knowledge and common knowledge have become increasingly used in economics and game theory. Aumann (1976) was the first economist ${ }^{3}$ to define common knowledge technically in order to prove that agents cannot "agree to disagree", i.e., they cannot disagree about their posterior probabilities if they are common knowledge. In other words, rational economic agents having common knowledge of their beliefs about an event cannot disagree. In terms of consensus this result means that a consensus about posteriors is achieved when these posteriors are common knowledge.

Several authors were interested in the way posteriors might become common knowledge. They proved the general convergence to identical and common knowledge messages when perfectly reliable communication takes place (i.e., when communication is not noisy and when there is no intertemporal uncertainty). First of all, Geanakoplos and Polemarchakis (1982) showed that when two agents exchange their posteriors about an event then the revision of these posteriors converge in a finite number of periods, and these probabilities become common knowledge, and hence equal. Roughly speaking, "we can't disagree forever". Thus, a consensus about posteriors is obtained during communication and this consensus becomes common knowledge. Later, Cave (1983) generalized this result to public communication with many agents when the function which determines sent messages is union consistent. ${ }^{4}$ Nevertheless, Parikh and Krasucki (1990) showed that for non-public, pairwise communication, union consistency is not strong enough to ensure the emergence of a consensus. Actually, they showed that if the function that determines sent messages is convex (which is a stronger requirement than union consistency), and if the communication protocol is "fair", then a consensus is always achieved. This work was pursued further

\footnotetext{
${ }^{2}$ Epistemic conditions for Nash equilibrium are sufficient conditions on knowledge for the realization of a Nash equilibrium. They were formally characterized by Aumann and Brandenburger (1995).

${ }^{3}$ Logical and formal analysis of knowledge really started in the sixties in philosophy, and was largely developed in cognitive science, linguistics, artificial intelligence, computer science, psychology, and game theory. For a survey see, e.g., Halpern (1995).

${ }^{4} \mathrm{~A}$ function $f: 2^{\Omega} \rightarrow \mathcal{M}$ is union consistent if $f(E)=f(F)$ and $E \cap F=\emptyset$ imply $f(E \cup F)=f(E)$. In other words, if an agent sends the same message at $E$ and $F$ then he sends the same message when he does not know which of these events realized. Posterior probabilities verify this property.
} 
by Krasucki (1990, 1996).

In their conclusion, Parikh and Krasucki (1990) claimed informally that common knowledge of sent messages is not always verified, despite consensus about them is always obtained. They argued that within their framework consensus is sometimes achieved without common knowledge of this consensus, i.e., without common knowledge of sent messages. This claim was contradicted by Weyers (1992) who showed, within the same framework, that sent messages always become common knowledge.

The intuition that the consensus is obtained but not necessarily common knowledge stems from computer science. This later field usually makes use of epistemic logic endowed with intertemporal uncertainties. That is, communication errors are possible, or there is no upper-bound for message delivery (see particularly Halpern and Moses (1990)). Besides, Heifetz (1996) gave an example of consensus without common knowledge in a setting which includes intertemporal uncertainty and where knowledge is represented by a partitional information structure on a state space. Thus, he showed that the standard game-theoretic setting permits to illustrate the phenomenon of consensus without common knowledge.

In this paper we consider the same knowledge framework as Heifetz (1996). We show that with noisy and non-public communication no event can become common knowledge if it was not so at the beginning. In other words, if communication is not public, common knowledge cannot be attained when perfect communication is not guaranteed. This result does not depend on the richness of language, i.e., is independent of communication details. It turns out that Heifetz's (1996) example, based on communication between three agents, is a particular case of our model. Moreover, we exhibit a simpler and minimal example that involves only two players. In our example, inspired by the electronic mail game ${ }^{5}$ (or coordinated attack problem), a consensus also emerges without common knowledge. A general result for the emergence of consensus without common knowledge is given for noisy, non-public and fair protocols.

For noisy and public communication we provide some results on the emergence of common knowledge and consensus which depend on the richness of the available language. Then, we apply our results to epistemic conditions for Nash equilibrium to show that if conjectures are not common knowledge, noisy and non-public communication protocols cannot make them common knowledge. Thus, even with truthful and mandatory reports, such a communication is not sufficient to achieve a mixed Nash equilibrium if there are more than two agents. However, with only two agents or with a noisy and public communication protocol, we give sufficient conditions for conjectures

\footnotetext{
${ }^{5}$ The electronic mail game was introduced by Rubinstein (1989). It is a 2-player coordination game with incomplete information where players communicate by noisy electronic mail signals.
} 
to form a Nash equilibrium in a finite number of communication periods.

We describe the model and some definitions in the next section. In section 3, we provide our results with some examples and illustrations. In section 4, we focus on Nash equilibrium by applying our model to noisy communication of conjectures. We conclude in section 5. Proofs are in appendix.

\section{The model}

\subsection{The basic framework}

We consider a set of agents with private information represented by a partitional information structure on a state space. An exogenous communication protocol determines sequential information exchanges between agents. Like Heifetz (1996), we incorporate time stages into the information structure. This is done by considering noisy communication between some agents at each period. By noisy communication we mean communication where the set of messages can fail to be delivered with a strictly positive probability at each period. Thus, agents are not always and immediately sure if the message they send at a certain period reaches the receivers at this period. In this case, they are not able to distinguish the current period from the preceding period. Therefore, such a setting generates intertemporal uncertainties.

The global state will correspond to the realization of a "basic" state completed with the number of periods during which messages have been transmitted. The probability of transmission failure at each period is strictly positive. Without loss of generality, we suppose that once an error occurs no message will be sent afterwards. We will say that we are at period $t$ when exactly $t$ waves of messages have been sent.

Formally, let $(\Omega, q)$ be a finite probability space with $q(\omega)>0$ for all $\omega \in \Omega$, and $(T, \mu)$ a probability space with $\mu(t)>0$ for any $t \in T \equiv \mathbb{N} \cup 0$, where $\mathbb{N}=\{1,2, \ldots\}$. We assume that $\mu(t)<\mu(t-1)$ for all $t \in \mathbb{N}$. Let $S=\Omega \times T$ be the space of states of the world with a probability measure $p$ such that $p(s)=q(\omega) \times \mu(t)$, where $s=(\omega, t) \in S$. A state $s=(\omega, t) \in S$ is a global state composed by the basic state $\omega \in \Omega$ and the time state $t \in T$.

Let $N=\{1, \ldots, n\}$ be the set of agents, with $n>1,\left(H_{i}\right)_{i \in N}$ their information partitions on $S, \mathbb{M}=H_{1} \wedge \ldots \wedge H_{n}$ the Meet (finest common coarsening) and $\mathbb{J}=H_{1} \vee \ldots \vee H_{n}$ the Join (coarsest common refinement) of these partitions. The sets $h_{i}(s), M(s)$ and $J(s)$ are respectively the elements of $H_{i}, \mathbb{M}$ and $\mathbb{J}$ containing $s \in S$. These sets are sufficient to describe the configuration of agents' knowledge and any order of interactive knowledge in the group. The set $h_{i}(s)$ contains global states that agent $i$ cannot distinguish when the real global state is $s$. Because time stages of $T$ are included in states of $S$, this information structure also describes how knowledge is distributed throughout time. In particular, it describes 
intertemporal uncertainties, i.e., it gives periods that each agent cannot distinguish.

When we are interested by consensus and by first-order knowledge about the states of $\Omega$ at a certain period, it is useful to represent agents' partitions that are only defined on $\Omega$. To this end, let $P_{\Omega}^{t}: 2^{S} \rightarrow 2^{\Omega}$ be the projection on $\Omega$ at $t$ such that for any $F \in 2^{S}$, we have $P_{\Omega}^{t}(F)=\{\omega \in \Omega:(\omega, t) \in F\}$. We denote by $h_{i}^{t}(\omega) \equiv P_{\Omega}^{t}\left(h_{i}(\omega, t)\right)$ the information set of player $i$ on $\Omega$ at period $t$. It contains states of $\Omega$ that player $i$ cannot distinguish from $\omega$ at period $t$. The partition generated by the sets $\left(h_{i}^{t}(\omega)\right)_{\omega \in \Omega}$ is denoted by $H_{i}^{t}$ for all $i \in N$ and $t \in T$. These partitions are sufficient to describe consensus and first-order knowledge but are not sufficient to describe agents' knowledge about others' knowledge. Indeed, if agents do not know in which period they are, they may be uncertain about others' information at the current period.

An event $E \subseteq \Omega$ will be called a basic event (or simply event if there is no possible confusion with events of $S$ ). For a set of agents $N^{\prime} \subseteq N$, let $J_{N^{\prime}}(\omega, t)$ be the element of $\bigvee_{i \in N^{\prime}} H_{i}$ containing $(\omega, t)$. The set $J_{N}(\omega, t)$ is the element of the Join of $\left(H_{i}\right)_{i \in N}$. We define $J_{N^{\prime}}^{t}(\omega) \equiv P_{\Omega}^{t}\left(J_{N^{\prime}}(\omega, t)\right)$ and $M^{t}(\omega) \equiv P_{\Omega}^{t}(M(\omega, t))$. We adapt interactive knowledge definitions to information structures incorporating time stages in the following subsection. ${ }^{6}$

\subsection{Intertemporal knowledge}

We say that a basic event $E$ is known at period $t$ by agent $i$ at $\omega \in \Omega$ if $E$ is realized at all states he considers possible at $(\omega, t)$, i.e., if $h_{i}^{t}(\omega) \subseteq E$. The event $E$ is mutually known at period $t$ if all agents know it at period $t$. These kinds of knowledge only include first-order knowledge because they do not involve interpersonal knowledge reasoning. Thus, they can be defined without considering the information structure over the whole state space $\Omega \times T$. Information partitions on $\Omega$ are sufficient to describe such knowledge. For interactive knowledge (as $c$-common knowledge or common knowledge) this is not sufficient. The basic event $E$ will be said $c$-common knowledge at period $t$ if everyone knows that everyone knows [ $c$ times] that everyone knows $E$ at $t$. It is common knowledge at $t$ if it is $c$-common knowledge at $t$ when $c$ goes to infinity. When $c$ is high but finite, $c$-common knowledge is also called "almost" common knowledge. Finally, the knowledge about $E$ will be called distributed among a set of agents at $t$ if this knowledge is acquired when agents perfectly share all their knowledge at period $t$.

Formally, let $K_{i}: 2^{S} \rightarrow 2^{S}$ be the knowledge operator of agent $i$. As usual, for any event $\tilde{E} \in 2^{S}, K_{i} \tilde{E}$ is the event "agent $i$ knows $\tilde{E}$ ". That is, the knowledge operator gives for each event the states in which the agent knows

\footnotetext{
${ }^{6}$ For a survey on interactive knowledge modeling in game theory see, e.g., Geanakoplos (1994).
} 
this event. To differentiate knowledge at different periods we introduce the following definitions.

Definition 1 Consider a basic event $E \subseteq \Omega$, a set of agents $N^{\prime} \subseteq N$, and suppose that the real state is $s=(\omega, t)$. Let $\tilde{E} \equiv\{(\omega, t) \in S: \bar{\omega} \in E\}=$ $E \times\{t\}_{t \in T}$.

- $E$ is known at period $\boldsymbol{t}$ by agent $i$, if $s \in K_{i} \tilde{E} \equiv\left\{s^{\prime} \in S: h_{i}\left(s^{\prime}\right) \subseteq\right.$ $\tilde{E}\}$, or equivalently if $h_{i}^{t}(\omega) \subseteq E$.

- $E$ is mutually known at period $\boldsymbol{t}$, if $s \in K \tilde{E} \equiv \bigcap_{i \in N} K_{i} \tilde{E}$, or equivalently if $\bigcup_{i \in N} h_{i}^{t}(\omega) \subseteq E$.

- $E$ is c-common knowledge at period t, if $s \in \underbrace{K \ldots K}_{c \text { times }} \tilde{E}=K^{c} \tilde{E}$.

- $E$ is common knowledge at period $\boldsymbol{t}$, if $M(s) \subseteq \tilde{E}$, or equivalently if $M^{t}(\omega) \subseteq E$ or if $s \in C K E \equiv K^{\infty} E$.

- The knowledge about $E$ is distributed among $N^{\prime}$ at period $t$, if $J_{N^{\prime}}^{t}(\omega) \subseteq E .^{7}$

\subsection{Communication protocols and consensus}

How knowledge is distributed during time is determined by a communication system $(f, P r)$. We define $P r$ as a noisy communication protocol, i.e., a function

$$
\begin{aligned}
\operatorname{Pr}: \mathbb{N} & \rightarrow\left(2^{N} \backslash\{\emptyset\}\right) \times\left(2^{N} \backslash\{\emptyset\}\right), \\
t & \mapsto(e(t), r(t)),
\end{aligned}
$$

which associates with probability $\frac{\mu(t)}{\mu(t-1)}$ to each period $t$ a set $e(t) \subseteq N$ of senders and a set $r(t) \subseteq N$ of receivers. ${ }^{8}$ With probability $1-\frac{\mu(t)}{\mu(t-1)}$ no message is sent at period $t$. Roughly speaking, a protocol determines who sends a message to whom, and in which order. The probability that exactly $t$ waves of messages have been transmitted is equal to $\mu(t) .{ }^{9}$ The function $f: 2^{\Omega} \rightarrow \mathcal{M}$, where $\mathcal{M}$ is an arbitrary set of messages, determines messages

\footnotetext{
${ }^{7}$ See Aumann (1976) and Nishihara (1991) for the proof of the equivalence between $M(s) \subseteq \tilde{E}$ and $s \in K^{\infty} \tilde{E}$. The other equivalences come from $s \in K_{i} \tilde{E} \Leftrightarrow h_{i}(\omega, t) \subseteq \tilde{E} \Leftrightarrow$ $h_{i}^{t}(\omega) \subseteq P_{\Omega}^{t} \tilde{E}=E$ and $M(\omega, t) \subseteq \tilde{E} \Leftrightarrow M^{t}(\omega) \subseteq P_{\Omega}^{t} \tilde{E}=E$.

${ }^{8} \mathrm{~A}$ protocol is often defined as a function from $\mathbb{N}$ to $N \times N$, i.e., it associates only one sender and one receiver at each period, as in Parikh and Krasucki (1990). Here, we consider rather a larger class of stochastic protocols that allow for several senders and several receivers at a time.

${ }^{9}$ For example, if the probability of transmission error at each period is constant and equal to $\varepsilon \in] 0,1\left[\right.$, then $\mu(t)=(1-\varepsilon)^{t} \varepsilon$ and $\frac{\mu(t)}{\mu(t-1)}=(1-\varepsilon)$.
} 
sent by each sender at each of his information set on $\Omega$. The characteristic of this function can be seen as the type of communication language used by agents.

Remark 1 The type of communication we consider in this paper is not strategic at all because the function $f$ which determines messages sent by agents is exogenous and imposed to them. When we will consider actions possibilities in section 4, communication should be seen as an exogenous procedure that imposes agents to truthfully communicate their conjectures.

Different kinds of relevant protocols can be considered. We will say that a communication protocol is fair if each agent can directly or indirectly send a message to any other agent an infinite number of times. In fair protocols, nobody is "excluded" from communication. A protocol is public if messages are sent simultaneously to all agents during at least one period. It is perfectly public when messages are always sent simultaneously from all agents to all the others. Formally:

Definition 2 A communication protocol $\operatorname{Pr}$ is:

- fair, if for all players $i, j \in N, i \neq j$, there exists an infinite number of finite sequences $t_{1}, \ldots, t_{K}$, with $t_{k} \in \mathbb{N}$ for all $k \in\{1, \ldots, K\}$, such that $i \in e\left(t_{1}\right)$ and $j \in r\left(t_{K}\right)$;

- $\boldsymbol{p u b l i c}$, if there exists $t \in \mathbb{N}$ such that $r(t)=N$;

- perfectly public, if $r(t)=e(t)=N$ for all $t \in \mathbb{N}$.

Clearly, a perfectly public protocol is fair and public. A public (fair) protocol can be fair (public), but not necessarily. Some particular protocols are given in the following example.

Example 1 (Some protocols) Let $N=\{1, \ldots, n\}$ be a set of agents, with $n \geq 2$.

1. Fair and non-public protocols: for all $t \in \mathbb{N}, e(t)=t \bmod n$ and $r(t)=t+1 \bmod n$.

2. Public and non-fair protocols: let $k \in N$ and consider two different agents $i$ and $j$. For all $t \leq k, e(t)=t$ and $r(t)=N$; for all $t>k$, $e(t)=N_{-i}$ and $r(t)=N_{-j}$.

3. Fair and public protocols:

- Let $k \in N$. For all $t \leq k, e(t)=t$ and $r(t)=N$; for all $t>k$, $e(t)=t \bmod n$ and $r(t)=t+1 \bmod n$.

- For all $t \geq 1, e(t)=t \bmod n$ and $r(t)=N$. 
We will say that a consensus about $f$ is obtained at period $t$ when agents agree about the value of $f$ at period $t$. Formally:

Definition 3 A consensus about $f$ is achieved at $(\omega, t) \in S$ if $f\left(h_{i}^{t}(\omega)\right)=$ $f\left(h_{j}^{t}(\omega)\right)=\bar{f}^{t}$ for all $i, j \in N$, or equivalently if $\omega \in\left[\bar{f}^{t}\right] \equiv\left\{\omega^{\prime} \in \Omega\right.$ : $\left.f\left(h_{j}^{t}\left(\omega^{\prime}\right)\right)=f\left(h_{i}^{t}(\omega)\right)=\bar{f}^{t} \forall i, j \in N\right\}$.

By definition 3 and by the definition of common knowledge, a consensus at $(\omega, t)$ is common knowledge at $(\omega, t)$ if $M^{t}(\omega) \subseteq\left[\bar{f}^{t}\right]$.

\subsection{The learning process}

At this stage, we do not specify any condition on information partitions on $S=\Omega \times T$. Because $f$ is only defined on $2^{\Omega}$, all what can be learned between agents are events of $\Omega$. Their knowledge about the events of $T$ will only be determined by the protocol itself. At the beginning of the process agents' knowledge about basic states of $\Omega$ is given by an initial information structure $\left(H_{i}^{0}\right)_{i \in N}$ on $\Omega$.

Let $\mathcal{M}^{n}$ be the Cartesian product of each agent's message space. Denote by $f^{t}(\omega) \in \mathcal{M}^{n}$ the vector of sent messages at $t$, where $f\left(h_{l}^{t}(\omega)\right)$ is the $l$-th coordinate of $f^{t}(\omega)$ for all $l \in e(t)$. For all $l \notin e(t)$, the $l$-th coordinate of $f^{t}(\omega)$ does not depend on $\omega$. We follow Parikh and Krasucki (1990) and we extend their learning process to our framework. For all $\omega \in \Omega$ and $t \in \mathbb{N}$ we have,

$$
\begin{aligned}
& h_{i}^{t}(\omega)=h_{i}^{t-1}(\omega) \cap\left\{\omega^{\prime} \in \Omega: f^{t}\left(\omega^{\prime}\right)=f^{t}(\omega)\right\}, \forall i \in r(t), \\
& \text { and } \quad h_{i}^{t}(\omega)=h_{i}^{t-1}(\omega), \forall i \notin r(t) .
\end{aligned}
$$

This learning process means that when player $i$ receives the message $f^{t}(\omega)$ at $t$ (i.e., $\left.i \in r(t)\right)$ from all the senders at that time, he excludes from his preceding information all the states of $\Omega$ which are incompatible with this message. When player $i$ does not receive this message (i.e., $i \notin r(t)$ ), he keeps his preceding information $h_{i}^{t-1}(\omega)$ unchanged. We will assume that agents who do not receive the message of period $t$ do not observe whether it reaches the receivers at $t$ or not. However, when an agent receives a message at period $t$, he knows that the protocol did not fail at this period. That is, he knows that receivers of $r(t)$ correctly get all messages from senders of $e(t)$ at this period.

After having described our framework and definitions, we now turn to the presentation of our results.

\section{Common knowledge and consensus emergence}

In this section, we provide four general results on the evolution of knowledge and on the emergence of consensus when communication is done through 
noisy communication protocols. Proposition 1 is an impossibility result. It states that common knowledge cannot emerge when communication is noisy and non-public. Nevertheless, in proposition 2 we show that with a sufficiently rich language and with fair protocols a consensus always emerges in a finite number of periods and this consensus becomes $c$-common knowledge for any positive integer $c$. However, common knowledge of this consensus does not necessarily emerge. In proposition 3 we show that when communication is public, the knowledge that is distributed among senders during public revelation periods becomes common knowledge if the communication language is sufficiently rich. Finally, under such language conditions or with union consistency, proposition 4 states that if communication is perfectly public then sent messages become common knowledge and equal in a finite number of periods. Thus, consensus and common knowledge emerge.

First, we give some properties that characterize our information structure framework. The following lemma states that if communication is noisy then agents who do not receive a message at period $t$ cannot distinguish period $t$ from period $t-1 .{ }^{10}$ It implies that if at each period, at least one agent does not receive any message, players' information partitions overlap through time.

Lemma 1 For all $\omega \in \Omega, h_{i}(\omega, t-1)=h_{i}(\omega, t)$ iff $i \notin r(t)$.

Remark 2 Our formalism is compatible with Heifetz's (1996) framework where information sets on $S$ are defined by $h_{i}(\omega, t)=\left\{\left(\omega^{\prime}, t^{\prime}\right): \omega^{\prime} \in h_{i}^{t}(\omega)\right.$, agent $i$ can not tell between the stages $\left.t, t^{\prime}\right\}$.

The following lemma provides other properties of the information structure. The first one corresponds to perfect recall. The second allows to express the projection of the Join of some partitions over the whole state space $\Omega \times T$ as a function of players' information on $\Omega$.

Lemma 2 For all $\omega \in \Omega, t \in \mathbb{N}, i \in N$, and $N^{\prime} \subseteq N$, we have the following properties:

1. $h_{i}^{t}(\omega) \subseteq h_{i}^{t-1}(\omega), M^{t}(\omega) \subseteq M^{t-1}(\omega)$ and $J_{N^{\prime}}^{t}(\omega) \subseteq J_{N^{\prime}}^{t-1}(\omega)$;

2. $J_{N^{\prime}}^{t}(\omega)=\bigcap_{i \in N^{\prime}} h_{i}^{t}(\omega)$.

By the second property of lemma 2, the projection of the Join of $\left(H_{i}\right)_{i \in N}$ is equal to the Join of the projection of these partitions. This property is not true for the Meet. More precisely, remind that $H_{i}^{t}$ is the partition of $\Omega$ generated by the sets $\left(h_{i}^{t}(\omega)\right)_{\omega \in \Omega}$. The Meet of $\left(H_{i}^{t}\right)_{i \in N}$ is denoted by $\mathbb{M}^{t} \equiv$ $\bigwedge_{i \in N} H_{i}^{t}$. Let $\bar{M}^{t}(\omega)$ be the element of $\mathbb{M}^{t}$ containing $\omega$. In general, with

\footnotetext{
${ }^{10}$ Lemma 1 is a direct implication of our time stages framework and as such, might be seen as an assumption.
} 
noisy communication protocols we have $\bar{M}^{t}(\omega) \neq M^{t}(\omega)$. This is illustrated in the following example, due to Heifetz (1996). In all examples, players' information sets are represented by the boxes depicted in figure 1. Player 1's information sets are represented by dotted boxes, player 2's information sets by solid boxes, and player 3's information sets by dashed boxes.

Player 1's information sets,

Player 2's information sets,

Player 3's information sets.

Figure 1: Players' information sets.

Example 2 (Heifetz, 1996) Suppose that $n=3, \Omega=\left\{\omega_{1}, \omega_{2}\right\}, p(\omega)=$ $1 / 2$ for all $\omega \in \Omega, H_{1}^{0}=\left\{\left\{\omega_{1}\right\},\left\{\omega_{2}\right\}\right\}, H_{2}^{0}=H_{3}^{0}=\{\Omega\}, r(t)=\{t, t+$ $1\} \bmod 3$ and $e(t)=t \bmod 3$. Such protocol corresponds to the situation where three agents are around a table. At each period, an agent sends a message to the agent at his left. The latter sends a message to the agent at his left in the next period, and so on. Agents who communicate at each period observe the reception of the message but the third agent does not. It is a fair and non-public protocol. The space $\Omega$ corresponds to the outcomes of a toss of a fair coin. Agent 1 is the only one who knows the real basic state.

Let $f(E)=p\left(\omega_{1} \mid E\right)$ and suppose that the real basic state is $\omega_{1}$. The information structure is represented by figure 2 on the next page. From the learning process (1) we clearly get a consensus at $t \geq 2$ because $f\left(h_{i}^{t}\left(\omega_{1}\right)\right)=$ 1 for all $i \in N$ and $t \geq 2$. This consensus at $t \geq 2$ corresponds to the basic event $\left\{\omega_{1}\right\}$. This event is not common knowledge at period 0 (because $\left.M^{0}\left(\omega_{1}\right)=\Omega \nsubseteq\left\{\omega_{1}\right\}\right)$ and it will never be common knowledge (because $M^{t}\left(\omega_{1}\right)=\Omega \nsubseteq\left\{\omega_{1}\right\}$ for all $\left.t \in \mathbb{N}\right)$. More generally, for all $\omega \in \Omega$ we have $M^{t}(\omega)=M^{0}(\omega)=\Omega$ for all $t \in \mathbb{N}$ and $\bar{M}^{t}(\omega)=\{\omega\}$ for all $t \geq 2$.

In the previous example common knowledge never emerges. We show in the following proposition that this impossibility is general: common knowledge can never evolve during noisy and non-public communication for any function $f$, i.e., for any available language.

Proposition 1 Consider a non-public and noisy communication protocol. If a (basic) event $E \subseteq \Omega$ is not common knowledge at a certain period $\bar{t} \in T$, it will never be common knowledge. 


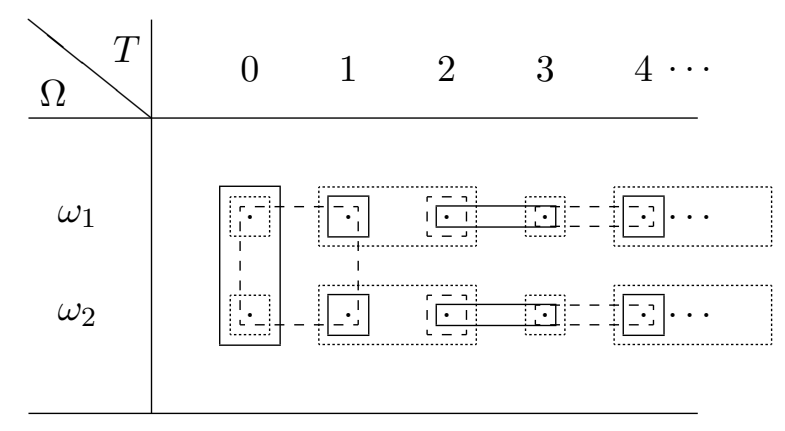

Figure 2: Heifetz's example.

The intuition of this result is that, when communication is non-public, there is at least one agent at each period who does not know if messages reach correctly the receiver(s) at this period. Therefore, any collective uncertainty that was present at the beginning is reachable when we apply iteratively a sufficiently long reasoning on interactive knowledge. This means that nothing that was not common knowledge can become common knowledge.

The following example shows the same phenomenon as Heifetz's example (i.e., it shows that a consensus is achievable without common knowledge), but involves only two agents.

Example 3 (A minimal example) Suppose that $n=2, \Omega=\left\{\omega_{1}, \omega_{2}\right\}$, $p(\omega)=1 / 2$ for all $\omega \in \Omega, H_{1}^{0}=\left\{\left\{\omega_{1}\right\},\left\{\omega_{2}\right\}\right\}, H_{2}^{0}=\{\Omega\}, e(t)=t \bmod 2$, $r(t)=t+1 \bmod 2$ and $f(E)=p\left(\omega_{1} \mid E\right)$. As in example 2, this protocol is fair and non-public.

Suppose that the real state is $\omega_{1}$. Clearly, a consensus about $f$ is obtained at $t \geq 1$, all players communicating the message "1" because $f\left(h_{i}^{t}\left(\omega_{1}\right)\right)=1$ for all $i \in N$ and $t \geq 1$. However, as before, the basic event $\left\{\omega_{1}\right\}$ which corresponds to this consensus at $t \geq 1$ will never be common knowledge. The information structure is represented by figure 3 on the following page. For all $\omega \in \Omega$ we have $M^{t}(\omega)=M^{0}(\omega)=\Omega$ for all $t \in \mathbb{N}$ and $\bar{M}^{t}(\omega)=\{\omega\}$ for all $t \geq 1$.

Why is publicity so essential? In particular, why do agents need to send messages to themselves for an eventual common knowledge creation? In fact, with perfect recall and perfectly reliable communication agents do not need to send messages to themselves. On the one hand, such a communication can be a tool to avoid absentmindedness. On the other hand, with noisy communication it is a tool for interactive knowledge creation. It helps agents to know if the messages they send at a certain period reach the receivers, and then to know who knows. This is necessary to generate common knowledge and is impossible if communication is noisy and non-public. 


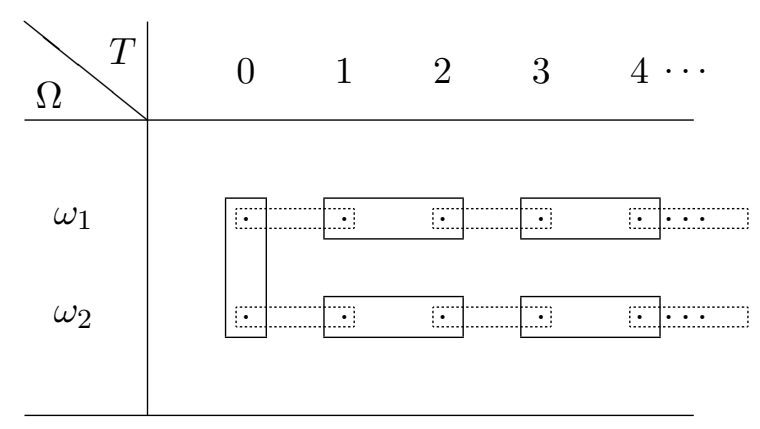

Figure 3: A minimal example.

A noisy and non-public communication does not exclude learning because proposition 1 allows to have $h_{i}^{t}(\omega) \nsubseteq F$ and $h_{i}^{t+1}(\omega)=F$ for some $F \subseteq \Omega$, as it was illustrated by examples 2 and 3 . Some learning occurred and a consensus was even achieved. Thus, in these examples, non-public communication was sufficient to achieve a consensus and also arbitrary high levels of interactive knowledge. Then, "almost" common knowledge states are achievable. The following proposition shows this general phenomenon for any fair protocol. This is obtained with an injectivity condition on the function $f$, which means that different information always gives different messages.

Formally, a function $f: 2^{\Omega} \rightarrow \mathcal{M}$ is injective on $2^{\Omega}$ if $E \neq F$ implies $f(E) \neq f(F)$, where $E, F \subseteq 2^{\Omega}$. Injectivity requires a relatively large set of possible messages $\mathcal{M}$, i.e., a relatively rich language. In previous examples, the function $f$ was injective. Let $Z$ be the set of basic events which are in an event of the initial Meet on $\Omega$, and which include one event of the Join on $\Omega$. Formally, $Z=\left\{z \subseteq 2^{\Omega}: \exists \omega \in \Omega\right.$ s.t. $z \subseteq M^{0}(\omega)$ and $\left.J^{0}(\omega) \subseteq z\right\}$, where $J^{0}(\omega) \equiv J_{N}^{t}(\omega)=P_{\Omega}^{t}(J(\omega, t))$. ${ }^{11}$ Injectivity will be sufficient on $Z$ because information sets on $\Omega$ always belong to $Z$.

Proposition 2 Let $c \in \mathbb{N}$ be an arbitrary integer. Suppose that $f$ is injective on $Z$ and that $\operatorname{Pr}$ is fair (public or not). Then there exists a period $\bar{t} \in T$ such that messages are $c$-common knowledge and equal at any period $t \geq \bar{t}$.

Remark 3 We have not determined all sufficient conditions on $f$ and $\operatorname{Pr}$ that guarantee consensus. Nevertheless, some necessary conditions are given by Krasucki (1990) with a deterministic protocol $\operatorname{Pr}: N \rightarrow N$. They are trivially necessary here.

\footnotetext{
${ }^{11}$ The set $J_{N}^{t}(\omega)$ does not depend on $t$ because agents cannot learn more than what is distributed among all of them (see lemma 2 with $N^{\prime}=N$ ).
} 
Remark 4 The application $f$ being injective, $c$-common knowledge of sent messages implies obviously $c$-common knowledge of all distributed knowledge.

Even with noisy communication, if an information transmission is public, i.e., observed by all agents, all what senders know can become common knowledge as it is shown in proposition 3. The need for public communication confirms that common knowledge creation requires face-to-face communication, or at least awareness reflexivity between communicators.

Proposition 3 If $f$ is injective on $Z$ and $P r$ is public then there exists $\bar{t} \in \mathbb{N}$ such that distributed knowledge among $e(\bar{t})$ is common knowledge at period $t$ for all $t \geq \bar{t}$.

The last result is illustrated in the following example.

Example 4 (A public, non-fair protocol) Let $\Omega=\left\{\omega_{1}, \omega_{2}, \omega_{3}\right\}, n=2$, $e(1)=1, r(1)=N$, and $e(t)=2, r(t)=1$ for all $t>1$. This protocol corresponds to example 1 , case 2 , with $k=1$. Here, we have $H_{1}^{0}=$ $\left\{\left\{\omega_{1}, \omega_{2}\right\},\left\{\omega_{3}\right\}\right\}$ and $H_{2}^{0}=\left\{\left\{\omega_{1}\right\},\left\{\omega_{2}, \omega_{3}\right\}\right\}$. The information structure is represented by figure 4 . We see that the event $\left\{\omega_{3}\right\}$ becomes common knowledge, but not $\left\{\omega_{1}\right\}$ because $M^{t}\left(\omega_{3}\right)=\left\{\omega_{3}\right\}$ for all $t \geq 1$ but $M^{t}\left(\omega_{1}\right)=M^{t}\left(\omega_{2}\right)=\left\{\omega_{1}, \omega_{2}\right\} \nsubseteq\left\{\omega_{1}\right\}$ for all $t \geq 1$. We see that player 2 will never know that player 1 knows $\left\{\omega_{1}\right\}$ or $\left\{\omega_{2}\right\}$. A consensus is achieved (because information sets on $\Omega$ are the same after period 1) but it will never be $c$-common knowledge for $c \geq 2$ at $\omega_{1}$ or $\omega_{2}$. If $r(t)$ is equal to 2 instead of 1 , a consensus at $\omega_{1}$ or $\omega_{2}$ will never emerge, even if the protocol is public (but it is not fair).

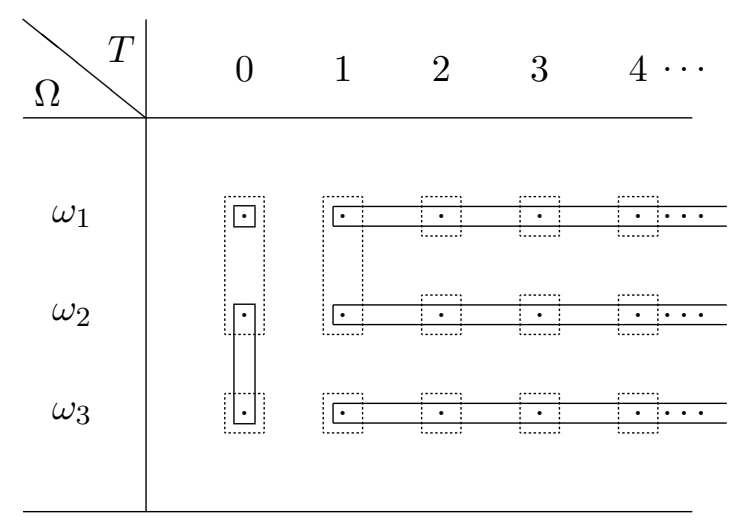

Figure 4: A public, non-fair protocol. 
The next proposition extends Cave's (1983) result to noisy and perfectly public communication. It states that consensus and common knowledge about this consensus are obtained in a finite number of periods for injective or union consistent ${ }^{12}$ functions if the protocol is perfectly public. All distributed knowledge does not necessarily become common knowledge because, in general, a union consistent function does not transmit all senders' information.

Proposition 4 If $f$ is union consistent or injective on $Z$ and $\operatorname{Pr}$ is perfectly public then messages become common knowledge and equal in a finite number of periods. ${ }^{13}$

Common knowledge and consensus emergence in perfectly public protocols is illustrated by the following example.

Example 5 (A perfectly public protocol) Consider the same initial information structure as in example 4, except that $H_{1}^{0}=\{\Omega\}$, with a perfectly public protocol, i.e., $e(t)=r(t)=N=\{1,2\}$ for all $t \in \mathbb{N}$. This information structure is represented by figure 5 . We see that all distributed knowledge becomes common knowledge at $t \geq 1$ because $J^{0}(\omega)=M^{t}(\omega)$ for all $t \geq 1$ and $\omega \in \Omega$.

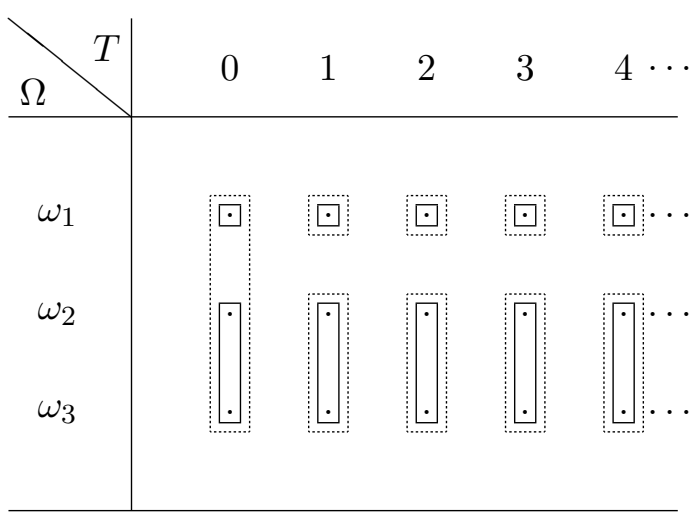

Figure 5: A perfectly public protocol.

The effect of noisy communication on strategic behaviors has already been analyzed in the electronic mail game, or coordinated attack problem. ${ }^{14}$ Actually, in this game, the reason for inefficiency is that common knowledge

\footnotetext{
${ }^{12}$ See footnote 4 on page 2 .

${ }^{13}$ Cave (1983) showed this result for non-noisy communication.

${ }^{14}$ See, e.g., Rubinstein (1989), Halpern and Moses (1990) and Morris and Shin (1997).
} 
is "good" at equilibrium but that common knowledge of the real game played is not obtained, because it was not common knowledge at the beginning and because of noisy and non-public communication. The communication protocol considered in the electronic mail game is exactly the one considered in example 3. Though, when communication is voluntary, we can obtain another communication protocol. In this case, common knowledge may not be necessary for coordination because there are equilibria where coordination occurs almost all the time. ${ }^{15}$ Thus, common knowledge is not necessarily a prerequisite for coordinated actions when communication is strategic. As it was shown in proposition 2 and in examples 2 and 3 it is also not always a prerequisite for agreement and consensus. ${ }^{16}$ In the next section, we will shed some light on the relationship between Nash equilibrium and common knowledge in order to apply our preceding results to this equilibrium concept. This is an application among many others that can be done in contexts where common knowledge is called upon.

\section{Application: noisy communication and Nash equi- librium}

In this section we will suppose that a basic state $\omega \in \Omega$ describes players' actions, payoff functions and initial beliefs (about others' actions, payoff functions and beliefs). The time states of $T$ will modify players' beliefs but not the other parameters. In particular, we do not allow agents to modify their actions at a given basic state even if their beliefs change at this state during time.

Aumann and Brandenburger (1995) formally showed that, in a game, if each player is rational, knows his own payoff function, and knows the others' strategy choices, then players' strategy choices constitute a Nash equilibrium of this game. This result applies for pure and for mixed strategies, if mixed strategies are viewed as conscious randomization. Thus, contrary to some general claims, common knowledge and interactive knowledge assumptions are not needed for Nash equilibrium. Only some mutual knowledge is needed.

Nevertheless, if we adopt the view of Nash equilibrium in terms of conjectures, common knowledge can be called upon. Indeed, if we suppose that agents choose a definite action, a mixture could represent players' uncertainties (conjectures) about a player's choice. In this case, common knowledge of those conjectures is a very weak sufficient condition for Nash equilibrium in $n$-player games when $n>2$. This will be stated formally in theorem 1 .

\footnotetext{
${ }^{15}$ See Binmore and Samuelson (1999).

${ }^{16}$ When common knowledge is required for agreement or coordination, Fagin, Halpern, Moses, and Vardi (1999) proposed to relax the granularity of time (to avoid intertemporal uncertainties) or the requirement for coordination.
} 
However, no other common knowledge assumptions (about payoff functions or players' rationality) are needed. In this section, we apply our results to such a setting.

First, suppose that we are at the initial period $t=0$. Let $A_{i}$ be the finite set of actions of player $i$. For all $\omega \in \Omega$, each player $i$ has a payoff function $\mathbf{u}_{i}(\omega): A \rightarrow \mathbb{R}$, where $A=\times_{i \in N} A_{i}$. The $n$-tuple of payoff functions $\mathbf{u}(\omega): A \rightarrow \mathbb{R}^{n}$ is called a $\boldsymbol{g a m e}$. Let $\mathbf{a}_{i}: \Omega \rightarrow A_{i}$ be the strategy of player $i$. A $n$-tuple of actions at $\omega$ is denoted by $\mathbf{a}(\omega)=\left(\mathbf{a}_{1}(\omega), \ldots, \mathbf{a}_{n}(\omega)\right)$. Given a $n$-tuple of actions $\mathbf{a}(\omega)$, i's payoff at $\omega$ is $\mathbf{u}_{i}(\omega)(\mathbf{a}(\omega))$. A conjecture $\phi_{i}(\omega)$ of player $i$ at $\omega$ is a probability distribution over $A_{-i}$. We write $[x]$ for the event $\{\omega \in \Omega: \mathbf{x}(\omega)=x\}$. Player $i$ 's conjecture about $\left[a_{-i}\right]$ at $\omega$ is defined by $\phi_{i}(\omega)\left(a_{-i}\right) \equiv p\left(\left[a_{-i}\right] \mid h_{i}^{0}(\omega)\right)$. A $n$-tuple of conjectures at $\omega$ is denoted by $\phi(\omega)=\left(\phi_{1}(\omega), \ldots, \phi_{n}(\omega)\right)$. For $j \neq i$, the conjecture of $i$ about $j$ induced by $\phi_{i}(\omega)$ is the marginal of $\phi_{i}(\omega)$ on $A_{j}$.

Definition 4 Player $i$ is rational at $\omega$ if

$$
E\left(\mathbf{u}_{i}(\cdot)\left(\mathbf{a}_{i}(\cdot), \mathbf{a}_{-i}(\cdot)\right) \mid h_{i}^{0}(\omega)\right) \geq E\left(\mathbf{u}_{i}(\cdot)\left(b_{i}, \mathbf{a}_{-i}(\cdot)\right) \mid h_{i}^{0}(\omega)\right), \forall b_{i} \in A_{i}
$$

Let $\left[R^{0}\right]$ be the set of states such that inequality (2) is verified for all players. Let $\bar{\omega}$ be the realized basic state. Let $u=\mathbf{u}(\bar{\omega})$ and $\phi=\phi(\bar{\omega})$ be respectively the game and the conjectures formed at $\bar{\omega}$, and suppose that agents are rational at $\bar{\omega}$, i.e., $\bar{\omega} \in\left[R^{0}\right]$. Aumann and Brandenburger (1995) proved the following result:

Theorem 1 (Aumann and Brandenburger, 1995) If $[u]$ and $\left[R^{0}\right]$ are mutually known and $[\phi]$ is commonly known at $\bar{\omega}$ then for each player $j$, all conjectures $\phi_{i}$ of players $i$ other than $j$ induce the same conjecture $\sigma_{j}$ about $j$, and $\left(\sigma_{1}, \ldots, \sigma_{n}\right)$ is a Nash equilibrium of $u$.

Remark 5 Theorem 1 does not state that common knowledge of the game is never important for Nash equilibrium. Indeed, mutual or higher-order knowledge of the game are sometimes not sufficient for some conjectures to be rational, when common knowledge may be sufficient. For example, in the electronic mail game, even if the game is "almost" common knowledge, a coordinated attack is not necessarily rational but it is with common knowledge of the game. ${ }^{17}$ Hence, rationality implies some knowledge assumptions, may imply some other assumptions of theorem 1 as well, and may imply even more.

Now, we suppose that players communicate through noisy protocols, as in the preceding sections. The game is the same at each period. During

\footnotetext{
${ }^{17}$ Common knowledge is however not necessary as it is shown with simpler communication protocols by Morris and Shin (1997).
} 
time, the game must not be seen as a repeated game. Agents communicate information on $\Omega$ through a given protocol. When we consider a certain period $t \in T$, we look at agents' beliefs and rationality at this period, as well as their conjectures to see if these last ones form a Nash equilibrium at this period.

We define the conjecture of player $i$ at $t$ by $\phi_{i}^{t}(\omega)\left(a_{-i}\right) \equiv p\left(\left[a_{-i}\right] \mid h_{i}^{t}(\omega)\right)$, where $h_{i}^{t}(\omega)$ follows the learning process defined by equation (1) on page 8 . Call player $i$ rational at $t$ if inequality (2) is verified by replacing " 0 " by " $t$ ". Let $\left[R^{t}\right]$ be the event "everybody is rational at $t$ ". A $n$-tuple of conjectures at $(\omega, t)$ is denoted by $\phi^{t}(\omega)=\left(\phi_{1}^{t}(\omega), \ldots, \phi_{n}^{t}(\omega)\right)$. Let $\phi^{t}=\phi^{t}(\bar{\omega})$ be the $n$-tuple of conjectures formed at $(\bar{\omega}, t)$.

In proposition 5 we apply proposition 1 to show that noisy and nonpublic communication between players is not sufficient to obtain a Nash equilibrium in a finite number of communication periods, even if the game and mutual rationality are mutually known.

Proposition 5 Let $\bar{t} \in T$ be a period. Suppose that $n>2$ and that $[u]$ and $\left[R^{\bar{t}}\right]$ are mutually known at $\bar{t}$ but that conjectures formed at $\bar{t}$ are not common knowledge at period 0 . Then, if the communication protocol is non-public and noisy, those conjectures do not necessarily form a Nash equilibrium of $u$ at $\bar{t}$.

Let $\varphi: 2^{\Omega} \rightarrow \mathcal{M}=\mathbb{R}^{n}$ be the application such that:

$$
\varphi(E)=\left(p\left(\left[a_{-1}\right] \mid E\right), \ldots, p\left(\left[a_{-n}\right] \mid E\right)\right), \forall E \subseteq \Omega .
$$

A direct application of proposition 4 gives the following result. It states that even if non-public and noisy communication protocols are not sufficient to achieve a Nash equilibrium, perfectly public ones might ensure a Nash equilibrium in a finite number of periods if the game is mutually known, and if agents' rationality is mutually known after a certain period.

Proposition 6 Consider a perfectly public communication protocol and suppose that there exists $t^{*} \in T$ such that $[u]$ and $\left[R^{t}\right]$ are mutually known at $t$ for all $t \geq t^{*}$. If $f=\varphi$ or if $f$ is injective on $Z$ then there exists a period $\bar{t} \geq t^{*}$ such that the conjectures at $\bar{t}$ form a Nash equilibrium of $u$.

Now, we prove that with only two agents, a noisy and non-public communication protocol is sufficient to ensure that agents' conjectures form a Nash equilibrium if their mutual rationality and the game are a distributed knowledge after a certain period. First, we introduce the following theorem:

Theorem 2 (Aumann and Brandenburger, 1995) Suppose that $n=$ 2. If $[u],\left[R^{0}\right]$ and $[\phi]$ are mutually known at $\bar{\omega}$ then $\left(\phi_{2}, \phi_{1}\right)$ is a Nash equilibrium of $u$. 
Theorem 2 and proposition 2 give the following result:

Proposition 7 Consider a fair (public or not) communication protocol. Suppose that $n=2$ and that there exists $t^{*} \in T$ such that the knowledge about $[u]$ and $\left[R^{t}\right]$ are distributed for all $t \geq t^{*}$. If $f$ is injective on $Z$ then there exists a period $\bar{t} \geq t^{*}$ such that $\left(\phi_{2}^{\bar{t}}, \phi_{1}^{\bar{t}}\right)$ is a Nash equilibrium of $u$.

\section{Concluding remarks}

Due to time imprecision, common knowledge creation was proved to be unlikely to occur in some practical settings. In this paper we have formally characterized different classes of communication protocols and communication languages that generate common knowledge or (and) consensus. We showed that intertemporal uncertainty combined with non-public communication prevent any common knowledge creation, whatever the available language is. Meanwhile, we also showed that some public announcements can generate common knowledge, and that fair communication allows for arbitrary high levels of interactive knowledge, depending on the available language. Even if standard common knowledge results obtained without intertemporal uncertainties do not hold, consensus and agreement results were shown to be easily achieved, however. Using these results, we showed that strong epistemic conditions for mixed Nash equilibrium (that are used for general $n$-player games) cannot be obtained with noisy and non-public communication, even if this communication is very precise and imposed on agents (i.e., knowledge sharing is mandatory). Nevertheless, for 2-player games or with public communication, sufficient conditions were provided to ensure the emergence of a Nash equilibrium in a finite number of communication periods.

In this paper, two important limitations were present: the lack of strategic (voluntary) communication, and excess cognitive rationality. ${ }^{18}$ Taking into account endogenous information structures or (and) cognitive irrationality is, to our mind, crucial for game theory. With noisy communication, this could be illustrated in the electronic mail game. Indeed, in this game, allowing strategic communication or limited rationality ${ }^{19}$ permits coordinated actions, which is the outcome we intuitively expect and that we observe in reality. Such phenomena should clearly be extended to many types of interactive decision making situations.

For general studies of endogenous information via communication a setting with noisy communication seems however difficult. To interpret voluntary disclosure of information agents need a clear temporality (as in meetings, face-to-face contacts, phone calls, ... ). They must distinguish the fact

\footnotetext{
${ }^{18}$ Cognitive rationality relies on the way agents represent and process information and beliefs.

${ }^{19}$ See respectively Binmore and Samuelson (1999) and Dulleck (1997).
} 
that an agent sends a message or does not send it in order to interpret communication choices. Technically, with noises, if we do not restrict the analysis to perfectly public communication, the partitions on which agents base their decisions and their choices of disclosure are never common knowledge, as it was shown in this paper. Advances in the analysis of strategic communication without noise, which is still not very well explored, may help to study the effects of the introduction of communication noises on incentives to communicate.

\section{Appendix: Proofs}

Proof of lemma 1. By $\mu(t)>0$ for all $t \in T$ and $q(\omega)>0$ for all $\omega \in \Omega$, we get $p(s)=q(\omega) \times \mu(t)>0$ for all $s \in S$. As each state of the world occurs with strictly positive probability, there is no null event, and thus we have $s \in h_{i}\left(s^{\prime}\right)$ iff $p\left(s \mid h_{i}\left(s^{\prime}\right)\right)>0$. Because agent $i$ does not receive the message at $t$ and because $\frac{\mu(t)}{\mu(t-1)}>0$, he assigns strictly positive probability that it does not reach the receivers, that is $p\left((\omega, t-1) \mid h_{i}(\omega, t)\right)>0$. Then, $(\omega, t-1) \in h_{i}(\omega, t)$. By the properties of partitional information structures, we get $h_{i}(\omega, t-1)=h_{i}(\omega, t) .{ }^{20}$

The converse is similar. If an agent receives a message at $t$ he knows that at least $t$ messages have been sent. Thus, if $i \in r(t)$, we have $p((\omega, t-$ 1) $\left.\mid h_{i}(\omega, t)\right)=0$. It follows that $(\omega, t-1) \notin h_{i}(\omega, t)$ and then $h_{i}(\omega, t-1) \neq$ $h_{i}(\omega, t)$.

Proof of lemma 2.

1. Directly from the learning process (1) which implies perfect recall.

2. The elements of the Join of some partitions are given by $J_{N^{\prime}}(s)=$ $\bigcap_{i \in N^{\prime}} h_{i}(s)$. Afterwards, notice that for $E, F \subseteq S$, we have $P_{\Omega}^{t}(E \cap F)=$ $P_{\Omega}^{t}(E) \cap P_{\Omega}^{t}(F)$. Thus, we get $J_{N^{\prime}}^{t}(\omega) \equiv P_{\Omega}^{t}\left(J_{N^{\prime}}(s)\right)=P_{\Omega}^{t}\left(\bigcap_{i \in N^{\prime}} h_{i}(s)\right)=$ $\bigcap_{i \in N^{\prime}} P_{\Omega}^{t}\left(h_{i}(s)\right) \equiv \bigcap_{i \in N^{\prime}} h_{i}^{t}(\omega)$.

Proof of proposition 1. Because the communication protocol is not public, for all $t \in \mathbb{N}$ there exists $i(t) \in N$ s.t. $i(t) \notin r(t)$. Thus, lemma 1 gives immediately $h_{i(t)}(\omega, t-1)=h_{i(t)}(\omega, t)$ for all $\omega \in \Omega$ and $t \in \mathbb{N}$. We obtain $M(\omega, t)=M\left(\omega, t^{\prime}\right)$ for all $t, t^{\prime} \in T .^{21}$ If $E$ is not common knowledge at $\bar{t}$, we have $M(\omega, \bar{t}) \nsubseteq \tilde{E}$, which gives immediately $M(\omega, t) \nsubseteq \tilde{E}$ for all $t \in T$, i.e., $E$ is never common knowledge.

Proof of proposition 3. Let $\bar{t}$ be the first period s.t. $r(\bar{t})=N$. This period exists because $\operatorname{Pr}$ is public. We get $h_{i}(\omega, k) \neq h_{i}(\omega, \bar{t})$ for all $i \in r(\bar{t})=N$

\footnotetext{
${ }^{20}$ With partitional information structures we have $s^{\prime} \in h_{i}(s)$ iff $h_{i}\left(s^{\prime}\right)=h_{i}(s)$.

${ }^{21}$ Think about the "reachability" criteria (Aumann (1976)): $\omega^{\prime}$ is reachable from $\omega$ in $\left(\Omega,\left(H_{i}\right)_{i \in N}\right)$ if there is a sequence of information sets $\left\langle h_{1}, h_{2}, \ldots, h_{k}\right\rangle$ s.t. $\omega \in h_{1}, \omega^{\prime} \in h_{k}$, where consecutive $h_{j}$ intersect and belong alternatively to some partitions of $\left(H_{i}\right)_{i \in N}$.
} 
and $k<\bar{t}$ by lemma 1 . It follows that:

$$
(\omega, k) \notin M(\omega, \bar{t}), \forall k<\bar{t} .
$$

Because $f$ is injective on $Z$, we have $f^{\bar{t}}\left(\omega^{\prime}\right)=f^{\bar{t}}(\omega)$ iff $h_{j}^{\bar{t}}(\omega)=h_{j}^{\bar{t}}\left(\omega^{\prime}\right)$ for all $j \in e(\bar{t})$, those last information sets always being included in $Z$. From the learning process defined by (1), we get:

$$
\begin{aligned}
\forall i \in N, \forall \omega & \in \Omega, \\
h_{i}^{\bar{t}}(\omega) & =h_{i}^{\bar{t}-1}(\omega) \cap\left\{\omega^{\prime} \in \Omega: h_{j}^{\bar{t}}(\omega)=h_{j}^{\bar{t}}\left(\omega^{\prime}\right), \forall j \in e(\bar{t})\right\}, \\
& =h_{i}^{\bar{t}-1}(\omega) \bigcap_{j \in e(\bar{t})} h_{j}^{\bar{t}}(\omega), \\
& \subseteq \bigcap_{j \in e(\bar{t})} h_{j}^{\bar{t}}(\omega)=J_{e(\bar{t})}^{\bar{t}}(\omega) \text { by lemma } 2 .
\end{aligned}
$$

This last event is the knowledge distributed among $e(\bar{t})$ at $(\omega, \bar{t})$. Because $\left(J_{e(\bar{t})}^{\bar{t}}(\omega)\right)_{\omega \in \Omega}$ forms a partition of $\Omega$, it is a common coarsening of the partitions $\left(H_{i}^{\bar{t}}\right)_{i \in N}$ by $(4)$. Thus, $\bar{M}^{\bar{t}}(\omega) \subseteq J_{e(\bar{t})}^{\bar{t}}(\omega)$ because $\overline{\mathbb{M}}^{t}$ is the finest common coarsening of the partitions $\left(H_{i}^{\bar{t}}\right)_{i \in N}$.

Now, we prove that $M^{\bar{t}}(\omega) \subseteq \bar{M}^{\bar{t}}(\omega)$. Set $\omega^{\prime} \in M^{\bar{t}}(\omega)$. Thus, $\left(\omega^{\prime}, \bar{t}\right) \in$ $M(\omega, \bar{t})$. From the "reachability" criteria, there exists a sequence,

$$
\left\langle h_{i_{1}}, \ldots, h_{i_{K}}\right\rangle=\left\langle h_{i_{1}}\left(\omega_{1}, t_{1}\right), \ldots, h_{i_{K}}\left(\omega_{K}, t_{K}\right)\right\rangle,
$$

with $i_{j} \in N, \omega_{j} \in \Omega, t_{j} \in T$ for all $j \in\{1, \ldots, K\}$ and $\omega_{1}=\omega^{\prime}, \omega_{K}=$ $\omega, t_{1}=t_{K}=\bar{t}$, where consecutive $h_{i_{j}}$ intercept. Consider the associate following sequence of information sets on $\Omega$ :

$$
\left\langle P_{\Omega}^{t_{1}} h_{i_{1}}\left(\omega_{1}, t_{1}\right), \ldots, P_{\Omega}^{t_{K}} h_{i_{K}}\left(\omega_{K}, t_{K}\right)\right\rangle=\left\langle h_{i_{1}}^{\bar{t}}\left(\omega^{\prime}\right), \ldots, h_{i_{k}}^{t_{k}}\left(\omega_{k}\right), \ldots, h_{i_{K}}^{\bar{t}}(\omega)\right\rangle .
$$

Those consecutive sets obviously intercept. By relation (3) we have $t_{j} \geq \bar{t}$ for all $j \in\{1, \ldots, K\}$. If not, consecutive $h_{i_{j}}$ of the initial sequence (5) would not intercept. Thus, by lemma 2 we have $h_{i_{j}}^{t_{j}}\left(\omega_{j}\right) \subseteq h_{i_{j}}^{\bar{t}}\left(\omega_{j}\right)$ for all $j \in\{1, \ldots, K\}$. Then, we can consider the following sequence of intercepting sets of $\Omega$ :

$$
\left\langle h_{i_{1}}^{\bar{t}}\left(\omega^{\prime}\right), \ldots, h_{i_{k}}^{\bar{t}}\left(\omega_{k}\right), \ldots, h_{i_{K}}^{\bar{t}}(\omega)\right\rangle .
$$

This proves that $\omega$ is reachable from $\omega^{\prime}$ through $\left(\Omega,\left(H_{i}^{\bar{t}}\right)_{i \in N}\right)$, i.e., $\omega^{\prime} \in$ $\bar{M}^{\bar{t}}(\omega)$. Thus, $M^{\bar{t}}(\omega) \subseteq \bar{M}^{\bar{t}}(\omega)$.

It follows that $M^{\bar{t}}(\omega) \subseteq J_{e(\bar{t})}^{\bar{t}}(\omega)$, i.e., all distributed knowledge among $e(\bar{t})$ at $\bar{t}$ is common knowledge at $t \geq \bar{t}$.

Proof of proposition 2. Let $\omega$ be the real state and set $\left[f^{t}\right]=\left\{\omega^{\prime} \in \Omega\right.$ : $\left.f\left(h_{j}^{t}\left(\omega^{\prime}\right)\right)=f\left(h_{i}^{t}(\omega)\right) \forall i, j \in N\right\}$. Because $f$ is injective, the learning process (1) gives (see the proof of proposition 3) $h_{i}^{t}(\omega) \subseteq h_{j}^{t}(\omega)$ for all 
$j \in e(t)$ and $i \in r(t)$. Thus, $\operatorname{Pr}$ being fair there exists $t_{1} \in \mathbb{N}$ s.t. $h_{i}^{t_{1}}(\omega) \subseteq$ $h_{j}^{t_{1}}(\omega)$, i.e., $h_{i}^{t_{1}}(\omega)=h_{j}^{t_{1}}(\omega)$ for all $i, j \in N .^{22}$ So, $h_{i}^{t_{1}}(\omega)=J^{0}(\omega)=\left[f^{t}\right]$ for all $i \in N$ and $t \geq t_{1}$, i.e., $\left[f^{t_{1}}\right]$ is mutually known at $t \geq t_{1}$. For $\widetilde{\left[f^{t_{1}}\right]}=\left\{\left(\omega^{\prime}, t^{\prime}\right) \in S: \omega^{\prime} \in\left[f^{t_{1}}\right]\right\}$ we get $\left[f^{t_{1}}\right] \times\{t\}_{t \geq t_{1}} \subseteq K\left[\widetilde{f^{t_{1}}}\right]$. Now, set $t_{2} \in \mathbb{N}$ s.t. for all $j \in N$, there exists $t^{\prime} \in\left[t_{1}, t_{2}\right]$ s.t. $j \in r\left(t^{\prime}\right)$. The period $t^{\prime}$ exists because $\operatorname{Pr}$ is fair. By lemma 1 , we get $h_{j}\left(\omega, t_{2}\right) \neq h_{j}(\omega, t)$ for all $t<t_{1}$ and $j \in N$. Thus, $h_{j}(\omega, t) \subseteq K \widetilde{\left[f^{t_{1}}\right]}$ for all $j \in N$ and $t \geq t_{2}$. This gives $\left[f^{t_{1}}\right] \times\{t\}_{t \geq t_{2}} \subseteq K K\left[\widetilde{\left.f^{t_{1}}\right]}\right.$. We easily see that we can continue the same reasoning any finite number of times to show that there exists $t_{c} \in \mathbb{N}$ s.t. $\left[f^{t_{1}}\right] \times\{t\}_{t \geq t_{c}} \subseteq K^{c} \widetilde{\left[f^{t_{1}}\right]}$. Then, there exists a period $t_{c}$ s.t. $\left(\omega, t_{c}\right) \in K^{c} \widetilde{\left[f^{t_{1}}\right]}$ (remind that $\omega \in\left[f^{t_{1}}\right]$ ), i.e., messages are $c$-common knowledge and equal (equality is already verified at time $t_{1}$ ). This completes the proof.

Proof of proposition 4. By the same reasoning as in the proof of proposition 3, if $\operatorname{Pr}$ is perfectly public we get $M^{t}(\omega) \subseteq \bar{M}^{t}(\omega)$ for all $\omega \in \Omega$ and $t \in T$. So, with $f$ union consistent Cave's (1983) result directly applies because he showed that there exists $\bar{t} \in T$ s.t. $\bar{M}^{\bar{t}}(\omega) \subseteq\left[f^{\bar{t}}\right]=\left\{\omega^{\prime} \in \Omega\right.$ : $\left.f\left(h_{j}^{\bar{t}}\left(\omega^{\prime}\right)\right)=f\left(h_{i}^{\bar{t}}(\omega)\right) \forall i, j \in N\right\}$. The restriction on $Z$ does not change the proof because $f$ is never applied outside $Z$. When $f$ is injective, proposition 4 is a corollary of proposition 3 with $\bar{t}=1$ and $e(1)=N$.

Proof of proposition 5. By proposition 1 we know that $\left[\phi^{\bar{t}}\right]$ will never be common knowledge because it is not at $t=0$ and because of non-public and noisy communication. Thus, $\left[\phi^{\bar{t}}\right]$ cannot be common knowledge at $\bar{t}$ and a condition of theorem 1 is not verified (and the conditions of this theorem cannot be weakened, as it was proved by the authors).

Proof of proposition 6. First, we show that $\varphi$ is union consistent. Let $E, F \subseteq \Omega$ s.t. $E \cap F=\emptyset$ and suppose that $\varphi(E)=\varphi(F)$. We have:

$$
\begin{aligned}
\varphi(E \cup F) & =\left(\frac{p\left(\left[a_{-i}\right] \cap(E \cup F)\right)}{p(E \cup F)}\right)_{i \in N}, \\
& =\left(\frac{p\left(\left[a_{-i}\right] \cap E\right)+p\left(\left[a_{-i}\right] \cap F\right)}{p(E)+p(F)}\right)_{i \in N}, \\
& =\left(p\left(\left[a_{-i}\right] \mid E\right)\right)_{i \in N} .
\end{aligned}
$$

Thus, $\varphi$ is union consistent. Because $\operatorname{Pr}$ is perfectly public we know by proposition 4 that for all $t^{*} \in T$ there exists $\bar{t} \geq t^{*}$ s.t. $\left[\phi^{\bar{t}}\right]=\{\omega \in \Omega$ : $\left.\phi\left(h_{i}^{\bar{t}}(\omega)\right)=\phi\left(h_{j}^{\bar{t}}(\bar{\omega})\right), \forall i, j \in N\right\}$ becomes common knowledge at $(\bar{\omega}, \bar{t})$. Then, because $[u]$ and $\left[R^{\bar{t}}\right]$ are mutually known at $\bar{t}$, we know that $\phi^{\bar{t}}$ form a Nash equilibrium of $u$ at $\bar{t}$ by theorem 1 .

\footnotetext{
${ }^{22}$ For perfectly public or non-noisy protocols the proof is completed because in this case we get $M^{t_{1}}(\omega) \subseteq \bar{M}^{t_{1}}(\omega)=h_{i}^{t_{1}}(\omega)=\left[f^{t_{1}}\right]$ for all $i \in N$.
} 
Proof of proposition 7. By proposition 2 we know that there exists $\bar{t} \geq t^{*}$ s.t. $[u]$ and $\left[R^{\bar{t}}\right]$ become $c$-common knowledge at $\bar{t}$, and thus mutually known at $\bar{t}$. Because $h_{i}^{t}(\omega)=h_{j}^{t}(\omega)$ for all $t \geq \bar{t}, \omega \in \Omega$, and $i, j \in N$, the conjectures become $c$-common knowledge at $\bar{t}$. Then, $[u],\left[R^{\bar{t}}\right]$ and $\left[\phi^{\bar{t}}\right]$ are mutually known at $\bar{t}$, i.e., $\left(\phi_{2}^{\bar{t}}, \phi_{1}^{\bar{t}}\right)$ is a Nash equilibrium of $u$ by theorem 2 .

\section{References}

Aumann, R. J. (1976): "Agreeing to disagree," The Annals of Statistics, 4(6), 1236-1239.

Aumann, R. J., and A. Brandenburger (1995): "Epistemic conditions for Nash equilibrium," Econometrica, 63(5), 1161-1180.

Binmore, K., and L. Samuelson (1999): "Coordinated action in the electronic mail game," mimeo.

Cave, J. A. K. (1983): "Learning to agree," Economics Letters, 12, 147152.

Dulleck, U. (1997): "A note on the e-mail game - Bounded rationality and induction," mimeo, Humboldt University, Institute of Economic Theory.

Fagin, R., J. Y. Halpern, Y. Moses, and M. Y. Vardi (1999): "Common knowledge revisited," Annals of Pure and Applied Logic, 96, 89-105.

Geanakoplos, J. (1994): "Common knowledge," in Handbook of Game Theory, ed. by R. J. Aumann, and S. Hart, vol. 2, chap. 40, pp. 14371496. Elsevier Science B. V.

Geanakoplos, J., and H. M. Polemarchakis (1982): "We can't disagree forever," Journal of Economic Theory, 28, 192-200.

HALPERN, J. Y. (1995): "Reasoning about knowledge: a survey," in Handbook of Logic in Artificial Intelligence and Logic Programming, ed. by D. Gabbay, C. J. Hogger, and J. A. Robinson, vol. 4, pp. 1-34. Oxford University Press.

Halpern, J. Y., ANd Y. Moses (1990): "Knowledge and common knowledge in a distributed environment," Journal of the ACM, 37(3), 549-587.

Heifetz, A. (1996): "Comment on consensus without common knowledge," Journal of Economic Theory, 70, 273-277.

Krasucki, P. (1990): "Reaching consensus on decisions," in Theoretical Aspects of Reasoning about Knowledge, Proc. of the Third Conf., ed. by R. Parikh, pp. 141-150. Morgan Kaufmann, San Francisco. 
(1996): "Protocols forcing consensus," Journal of Economic Theory, $70,266-272$.

Morris, S., And H. S. ShIn (1997): "Approximate common knowledge and co-ordination: recent lessons from game theory," Journal of Logic, Language, and Information, 6, 171-190.

Nishinara, K. (1991): "A note on the equivalence of the two definitions of common knowledge," Mathematical Social Sciences, 21, 177-178.

Parikh, R., and P. Krasucki (1990): "Communication, consensus, and knowledge," Journal of Economic Theory, 52, 178-189.

Rubinstein, A. (1989): "The electronic mail game: strategic behavior under "almost common knowledge"," American Economic Review, 79(3), 385-391.

Weyers, S. (1992): "Three results on communication, information and common knowledge," CORE Discussion Paper 9228, Université catholique de Louvain. 